\title{
Cartographie des cancers de la cavité orale chez l'enfant
}

\author{
N. Akerzoul (Rabat), S. Chbicheb (Rabat)
}

Objectif : Étudier le profil épidémiologique et histopathologique des cancers oraux chez les enfants pris en charge au services d'hémato-oncologie pédiatrique, de stomatologie et de chirurgie maxillo-faciale à l'hôpital 20 Aout de Casablanca, et à l'hôpital des enfants de Rabat. Notre objectif est de définir l'importance de l'oncologie pédiatrique, en particulier celle affectant la cavité orale, et aussi pour décrire les cancers oraux chez les enfants, leurs fréquences et les leurs caractéristiques histopathologiques. MATÉRIEL ET MÉTHODES: Il s'agit d'une étude rétrospective de 126 enfants hospitalisés entre 2010 et 2013 dans le service d'hémato-oncologie pédiatrique, du département de stomatologie et de chirurgie maxillo-faciale, de l'hôpital 20 août de Casablanca et le département d'hémato-oncologie pédiatrique de l'hôpital des enfants de Rabat; dans lequel nous avons diagnostiqué un cancer confirmé de la cavité orale. RÉSULTATS: Dans notre échantillon, tous les groupes d'âge ont été touchés par le processus de la maladie, mais les âges entre [0-4] ans et entre [13-16] étaient les plus touchés avec une moyenne d'âge de 8 ans, et des extrêmes allant de 4 mois à 16 ans. Dans notre échantillon de population, nous avons noté une légère prédominance avec $50,7 \%$ des cas. Le lymphome non hodgkinien Burkitt était le type histologique le plus commun avec 35,2\% des cas. Les joues représentaient la localisation la plus fréquente avec $37,9 \%$ des cas, alors que le maxillaire représentait 19,7\% des cas. La chimiothérapie a été la thérapeutique exclusive la plus utilisée dans notre échantillon dans 67,6\% des cas. CONCLUSION: Les caractéristiques épidémiologiques, cliniques et pathologiques des cancers de la cavité orale dans notre population ne sont pas différents des données de la littérature. Cependant, les parents manquent de conscience et de diagnostic tardif de ces les lésions qui semblent être responsables du profil dramatique des cancers oraux chez les enfants de la population marocaine. 